\title{
Síndrome de Fitz-Hugh-Curtis en un paciente varón anciano. Caso y revisión de la literatura
}

\author{
Fitz-Hugh-Curtis syndrome in elderly male patient. Case and literature review
}

Miguel A. Freiria-Eiras* y Cristina Varela-Lamas

Servicio de Cirugía del Aparato Digestivo, Hospital HM Rosaleda, Santiago de Compostela, La Coruña, España

\begin{abstract}
Resumen
El síndrome de Fitz-Hugh-Curtis (FHCS) es la inflamación de la cápsula hepática sin afectación del parénquima asociada a una enfermedad pélvica inflamatoria. Hay muy pocos casos descritos en varones. El síntoma característico es el dolor abdominal en el cuadrante superior derecho, que hace que se confunda el cuadro con una enfermedad de la vía biliar. Son características las adherencias fuertes entre el diafragma y el hígado en forma de "cuerda de violín". Presentamos el caso de un varón de 81 años que se somete a una colecistectomía laparoscópica por pancreatitis de repetición. Durante la cirugía se encuentran las características adherencias en "cuerda de violín", que se seccionan. El paciente da positivo para anticuerpos contra Chlamydia trachomatis. Hay nueve casos descritos en la literatura de FHCS en varones. Este síndrome se confunde muchas veces con patología infecciosa biliar, lo que nos obliga a someter al paciente a una cirugía para realizar el diagnóstico cuando se encuentran las características adherencias. Si sospechamos la enfermedad mediante las pruebas complementarias, podemos intentar tratarla con antibióticos.
\end{abstract}

PALABRAS CLAVE: Colecistectomía. Fitz-Hugh-Curtis. Clamidia.

\begin{abstract}
Fitz-Hugh-Curtis syndrome (FHCS) is the inflammation of the hepatic capsule without affecting the parenchyma, which is associated with a pelvic inflammatory disease. There have been very few cases in men. The main symptom is abdominal pain in the right upper quadrant, which can be confused with a bile duct disorder. Strong violin string-like adhesions between the diaphragm and the liver are characteristic. In the study concerned, it is reported the case of an 81 year-old man who undergoes a laparoscopic cholecystectomy for recurrent pancreatitis. During surgery, the typical violin string-like adhesions are found and sectioned. The patient tests positive for Chlamydia trachomatis antibodies. Only nine cases in men have been reported in FHCS literature. This syndrome is frequently confused with infectious biliary tract disease, so the patient should undergo a surgery to diagnose when the characteristic adhesions are found. If the disease is suspected by the additional tests, it can be treated with antibiotics.
\end{abstract}

KEY WORDS: Cholecystectomy. Fitz-Hugh-Curtis. Chlamydia.

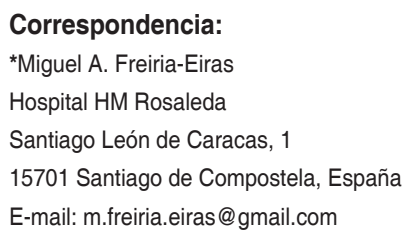

Fecha de recepción:15-03-2018

Fecha de aceptación: 16-06-2018

DOI: $10.24875 / C I R U .18000263$
Cir Cir. 2018;86:455-458

Contents available at PubMed www.cirugiaycirujanos.com 


\section{Introducción}

El síndrome de Fitz-Hugh-Curtis (FHCS) es un tipo de perihepatitis que causa la inflamación de la cápsula hepática sin afectar al parénquima. Este síndrome debe su nombre a Curtis, quien en $1930^{1}$ reportó la inflamación de la cápsula hepática con adherencias al diafragma en una paciente afectada de salpingitis; y a Fitz-Hugh, quien en $1934^{2}$ identificó al bacilo Neisseria gonorrhoeae como causante de adherencias entre el hígado y el diafragma en la característica forma de «cuerdas de violín».

Esta inflamación del hígado se cree que está provocada por la migración de patógenos por vía hemática o linfática desde un proceso infeccioso pélvico previo.

La mayoría de los casos de FHCS ocurren en mujeres jóvenes y en edad fértil diagnosticadas de enfermedad pélvica inflamatoria o de salpingitis aguda, acompañado de dolor en el cuadrante superior derecho del abdomen. La afectación en varones es muy infrecuente; solo se han reportado nueve casos en la literatura (Tabla 1).

Inicialmente, los dos microorganismos identificados con más frecuencia eran N. gonorrhoeae y Chlamydia trachomatis, pero actualmente se conocen otras bacterias de transmisión sexual causantes del proceso inflamatorio ${ }^{3}$.

El síntoma principal es el dolor en el cuadrante superior derecho del abdomen, que muchas veces lleva a confundir el FHCS con una enfermedad biliar, asociado a síntomas pélvicos inflamatorios en las mujeres. En los hombres, las manifestaciones pélvicas pueden estar ausentes.

En las pruebas de imagen puede encontrarse un realce de la cápsula hepática durante la realización de una tomografía computarizada (TC) abdominal con contraste intravenoso $0^{4,5}$.

A continuación presentamos el caso de un paciente varón de edad avanzada diagnosticado de FHCS durante una laparoscopia por patología biliar.

\section{Caso clínico}

Varón de 81 años que acude a urgencias por cuadro de dolor abdominal en mesogastrio, irradiado en cinturón, continuo, con distensión abdominal y náuseas, sin fiebre, ictericia ni coluria. Estable hemodinámicamente.

Antecedentes personales de hipertensión arterial, hipercolesterolemia, cistectomía parcial por cáncer de vejiga, enfermedad pulmonar obstructiva crónica y diverticulosis de colon sigmoide.

En el análisis de sangre encontramos 9800 leucocitos con un $79 \%$ de neutrófilos; el resto de hemograma y bioquímica normal, excepto una leve elevación de la amilasa, $125 \mathrm{U} / \mathrm{l}$ (23-85). Se realiza TC abdominal que revela pancreatitis aguda (Balthazar $\mathrm{C}$ ) en la cola del páncreas. En la colangiografía por resonancia magnética no se evidencia coledocolitiasis ni patología hepática; destacan la vesícula biliar con barro y un proceso inflamatorio en la cola del páncreas. El

Tabla 1. Casos publicados de síndrome de Fitz-Hugh-Curtis en varones

\begin{tabular}{|c|c|c|c|}
\hline Autores y año & Edad (años) & Presentación & Tratamiento \\
\hline Kimball y Knee, $1970^{13}$ & ND & ND & ND \\
\hline Francis y Osoba, $1972^{14}$ & 25 & Dolor abdominal y síntomas urinarios & Antibióticos \\
\hline Davidson y Hawkins, $1982^{15}$ & 35 & Dolor preural derecho, proctitis y uretritis & Antibióticos \\
\hline Baek, et al., $2010^{16}$ & 35 & Dolor en cuadrante derecho y síntomas urinarios & Antibióticos \\
\hline Saurabh, et al., $2010^{10}$ & 29 & Dolor en cuadrante derecho & Cirugía \\
\hline Rouhard, et al., 2014 12 & 45 & Dolor en cuadrante derecho & $\begin{array}{l}\text { Cirugía y } \\
\text { antibióticos }\end{array}$ \\
\hline Nardini, et al., $2015^{11}$ & 26 & Dolor abdominal y antecedentes de infección genital & Antibióticos \\
\hline Jeong, et al., $2015^{17}$ & ND & ND & ND \\
\hline Yi, et al., $2015^{6}$ & 60 & Dolor en cuadrante derecho & Antibióticos \\
\hline Freiria-Eiras, 2017 (actual) & 81 & Dolor en cuadrante derecho & $\begin{array}{l}\text { Cirugía y } \\
\text { antibióticos }\end{array}$ \\
\hline
\end{tabular}


paciente es tratado con medidas conservadoras y se da de alta al décimo día sin incidencias.

Una semana después, el paciente acude de nuevo a urgencias por el mismo dolor abdominal. En la analítica de sangre destacan unos valores de amilasa de $87 \mathrm{U} / \mathrm{l}$ y de lipasa de $813 \mathrm{U} / \mathrm{l}(0-160)$. En la TC abdominal aparece una lesión nodular en la cola del páncreas, sin datos de inflamación y líquido perihepático. El paciente se trata como un cuadro de pancreatitis aguda, tiene buena evolución y es dado de alta al duodécimo día.

Se planifica colecistectomía de forma programada, con abordaje laparoscópico. Tras la realización del neumoperitoneo se encuentran adherencias fuertes (Fig. 1) entre la cápsula hepática y el diafragma (en «cuerdas de violín»), características del FHCS. Se realiza colecistectomía y se seccionan las adherencias. En el posoperatorio es tratado con levofloxacino, $500 \mathrm{mg} / 12$ horas durante 7 días. El paciente se recupera sin incidencias y es dado de alta a las 24 horas tras la cirugía, con buena recuperación en su domicilio.

\section{Discusión}

Habitualmente nos referimos al FHCS como la inflamación de la cápsula hepática asociada a una inflamación pélvica, hecho que ocurre de forma más frecuente en las mujeres en edad reproductiva. Según Yi, et al. ${ }^{6}$, este síndrome puede dividirse en dos fases: una fase aguda en la que encontramos los característicos hallazgos de una inflamación exudativa de la cápsula hepática, con dolor abdominal agudo que puede irradiarse al hombro derecho; y una fase crónica en la que la inflamación del hígado y los exudados crean las adherencias del hígado al diafragma en la característica forma de "cuerdas de violín». Estas adherencias no deberían dar síntomas, pero en casos persistentes de dolor en el cuadrante superior derecho algunos autores, como Owens et al. ${ }^{7}$, recomiendan su sección.

En la mayoría de los casos no se llega al diagnóstico del microorganismo causante de la infección, ya que se suele tratar a los enfermos de forma empírica con antibióticos al sospechar una patología infecciosa biliar. La forma de diseminación del patógeno desde la zona pélvica aún no está clara, sobre todo en los varones, pues la mayoría no presentan síntomas infecciosos en los genitales.

Lo más importante para un correcto diagnóstico es la sospecha de la enfermedad por el médico. Este hecho muchas veces es difícil, ya que la mayoría de

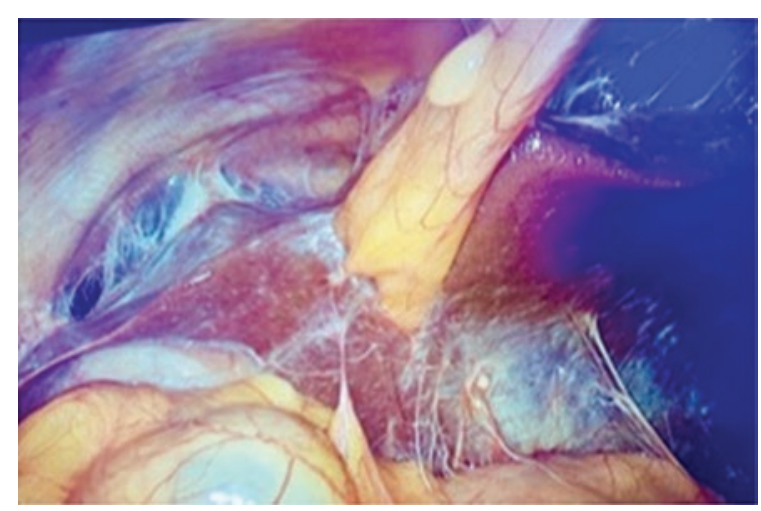

Figura 1. Características adherencias en «cuerdas de violín».

los casos en varones se confunden con patología biliar, como una colecistitis aguda, y muchas veces se realiza el diagnóstico en el mismo acto operatorio. Si se solicita una TC abdominal, un radiólogo con experiencia puede sospechar la perihepatitis si observa el realce de la cápsula hepática cuando realiza el estudio con contraste intravenoso en fase arterial. En nuestro caso no visualizamos dicho realce, pero sí se evidenció una colección líquida perihepática coincidiendo con el cuadro de dolor abdominal.

Si realizamos un diagnóstico certero de FHCS mediante la clínica del paciente y sus pruebas complementarias, podemos intentar un tratamiento con antibióticos empíricos sin necesidad de identificar el microorganismo causante ${ }^{8}$. Si a pesar de un tratamiento antibiótico correcto el dolor abdominal persiste, podemos intentar seccionar las adherencias creadas entre el hígado y el diafragma, como realizan Reichert y Valle?.

Como ya hemos comentado, este síndrome es muy raro en los varones. Hemos recogido los casos publicados en la literatura, que resumimos en la tabla 1, y seguidamente comentamos algunos de ellos.

Yi, et al. ${ }^{6}$ publicaron en 2015 el caso de un varón de 60 años con dolor en el cuadrante superior derecho y antecedente de gonorrea, diagnosticado por los síntomas y por los resultados de la TC abdominal.

Saurabh, et al..$^{10}$ publicaron en 2012 el caso de un paciente joven, de 29 años, con un episodio de dolor en los cuadrantes derechos, diarrea, náuseas y vómitos, a quien realizaron cirugía por persistencia del dolor sin encontrar la causa en las pruebas complementarias, y detectaron las características adherencias en "cuerda de violín» entre el hígado y el diafragma.

Nardini, et al. ${ }^{11}$ describen en 2014 el caso de un varón de 26 años que acude a urgencias por dolor abdominal en el cuadrante superior derecho, 
acompañado de dolor al orinary antecedente reciente de relaciones sexuales de riesgo. En la ecografía abdominal se observa una pequeña cantidad de líquido perihepático, y los test de exudado de uretra dan positivo para $C$. trachomatis y $N$. gonorrhoeae. El paciente fue tratado satisfactoriamente con antibióticos.

Por último, señalar el caso publicado en 2014 por Rouhard, et al. ${ }^{12}$, de un varón de 45 años con dolor crónico en el cuadrante superior derecho, a quien realizaron una laparoscopia exploradora y visualizaron las características adherencias en "cuerda de violín». El paciente fue tratado satisfactoriamente con antibióticos.

En nuestro caso hemos presentado un paciente de avanzada edad con un cuadro sugestivo de patología pancreática y biliar. Las pruebas de imagen no nos hicieron sospechar la posibilidad de un FHCS, y el diagnóstico se estableció tras la laparoscopia inicial para una colecistectomía. En sucesivas revisiones solicitamos serología para enfermedades de transmisión sexual, que fueron todas negativas, excepto para anticuerpos contra $C$. trachomatis. Interrogado el paciente, refiere no haber tenido nunca prácticas sexuales de riesgo, pero dice tener problemas urinarios desde hace 6 meses aproximadamente. No se ha encontrado la causa de la infección por $C$. trachomatis, pero sospechamos que este patógeno ha provocado los cuadros de dolor abdominal e inflamación que ha presentado el paciente.

\section{Conclusión}

El FHCS es un proceso infeccioso en el que se produce una perihepatitis a partir de una infección genitourinaria. Es más frecuente en el sexo femenino. Se han descrito pocos casos en varones. La imagen característica de la perihepatitis son la adherencias entre la cápsula hepática y el diafragma en "cuerdas de violín».

Si se sospecha este síndrome por la clínica del paciente y las pruebas de imagen, se recomienda tratamiento antibiótico dirigido hacia los microorganismos más frecuentes ( $N$. gonorrhoeae y $C$. trachomatis); en cambio, muchas veces se realiza el diagnóstico durante el acto operatorio por sospecha de patología biliar, al descubrir las características adherencias entre el hígado y el diafragma.

Nosotros presentamos un caso de FHCS en un varón de 81 años por $C$. trachomatis, en quien se encontraron las características adherencias entre el hígado y el diafragma tras una laparoscopia para realizar una colecistectomía por pancreatitis de repetición.

\section{Conflicto de intereses}

Los autores declaran no tener conflictos de intereses.

\section{Bibliografía}

1. Curtis $\mathrm{AH}$. A cause of adhesions in the right upper quadrant. JAMA. 1930;94:1221-2.

2. Fitz-Hugh $\mathrm{Jr} \mathrm{T}$. Acute gonococcic peritonitis of the right upper quadrant in women. JAMA. 1934;102: 2094-6.

3. Wang SP, Eschenbach DA, Holmes KK, Wager G, Grayston JT. Chlamydia trachomatis infection in Fitz-Hugh-Curtis syndrome. Am J Obstet Gynecol. 1980;138:1034-8.

4. Wang CL, Guo XJ, Yuan ZD, Shi Q, Hu XH, Fang L. Radiologic diagnosis of Fitz-Hugh-Curtis syndrome. Chin Med J (Engl). 2009;122:741-4.

5. Nishie A, Yoshimitsu K, Irie $H$, Yoshitake T, Aibe H, Tajima T, et al. Fitz-Hugh-Curtis syndrome. Radiologic manifestation. J Comput Assist Tomogr. 2003;27:786-91

6. Yi H, Shim CS, Kim GW, Kim JS, Choi IZ. Case of Fitz-Hugh-Curtis syndrome in male without presentation of sexually transmitted disease. World J Clin Cases. 2015;3:965-9.

7. Owens S, Yeko TR, Bloy R, Maroulis GB. Laparoscopic treatment of painful perihepatic adhesions in Fitz-Hugh-Curtis syndrome. Obstet Gynecol. 1991;78:542-3.

8. Peter NG, Clark LR, Jaeger JR. Fitz-Hugh-Curtis syndrome: a diagnosis to consider in women with right upper quadrant pain. Cleve Clin J Med. 2004;71:233-9.

9. Reichert JA, Valle RF. Fitz-Hugh-Curtis syndrome. A laparoscopic approach. JAMA. 1976;236:266-8.

10. Saurabh S, Unger E, Pavlides C. Fitz-Hugh-Curtis syndrome in a male patient. JSCR. 2012;3:12.

11. Nardini P, Compri M, Marangoni A, D'Antuono A, Bellavista S, Calvanese $C$, et al. Acute Fitz-Hugh-Curtis syndrome in a man due to gonococcal infection. J Emerg Med. 2015;48:e59-62.

12. Rouhard $S$, Maldague $P$, Ramboux A. Fitz-Hugh-Curtis syndrome in a man. Endoscopy. 2014;46 Suppl 1 UCTN: E1.

13. Kimball MW, Knee S. Gonococcal perihepatitis in a male. The Fitz-Hugh-Curtis syndrome. N Engl J Med. 1970;282:1082-4.

14. Francis TI, Osoba AO. Gonococcal hepatitis (Fitz-Hugh-Curtis syndrome) in a male patient. Br J Vener Dis. 1972;48:187-8.

15. Davidson AC, Hawkins DA. Pleuritic pain: Fitz Hugh Curtis syndrome in a man. Br Med J (Clin Res Ed). 1982;284:808.

16. Baek HC, Bae YS, Lee KJ, Kim DH, Bae SH, Kim DW, et al. A case of Fitz-Hugh-Curtis syndrome in a male. Korean $\mathrm{J}$ Gastroenterol. 2010;55:203-7.

17. Jeong TO, Song JS, Oh TH, Lee JB, Jin YH, Yoon JC. Fitz-Hugh-Curtis syndrome in a male patient due to urinary tract infection. Clin Imaging. 2015;39:917-9. 